\title{
EP-149
}

\section{First experience pure laparoscopic HPD for cholangiocarcinoma: The feasibility and safety of short term surgical outcomes}

\author{
Jin Ho LEE*, Jae Uk JEONG, Kuk Hwan KWON
}

Department of Surgery, National Health Insurance Service Ilsan Hospital, Goyang, Korea

Introduction: Recently, as laparoscopic surgery has developed, bile duct cancer surgery has also been performed with pancreaticoduodenectomy (PD) or liver resection using laparoscopic system. In some cases, bile duct cancer may require hepaticopancreaticoduodenectomy (HPD) surgery, which requires simultaneous liver Resection and PD to obtain an appropriate resection margin. In this study, we report the feasibility and safety of the pure laparoscopic HPD.

Methods: The patient was a 65-year-old male who underwent pure laparoscopic HPD surgery on October 16, 2019 in our hospital. The patient underwent surgery in the same trocar position as the conventional laparosocpic PPPD, and hepatectomy was performed for right hepatectomy. Since the patient was diagnosed with distal biliary tract cancer at the time of initial diagnosis, we used the same trocar position as laparosocpic PPPD and three 10-mm trocars and two 5-mm trocars. However, during surgery, a positive for malignancy was reported until the third frozen biopsy of the proximal bile duct margin. Therefore, right hepatectomy was performed to obtain a hepatic resection for distal bile duct margin.

Results: The operation time was 741 minutes, and the amount of bleeding was $750 \mathrm{~mL}$. And the length of hospital stay was 21 days due to postoperative ascites control. There was no major complication but postoperative ascites.

Conclusions: The pure laparoscopic HPD procedure is feasible and safety method though operation time takes a little longer. Therefore, laparoscopic surgeons who are familiar with laparoscopic surgery may try laparoscopic HPD as another treatment method. 